\title{
The effect of mass ratio on the flow characteristics of hydrogen- natural gas mixture using reduced order modelling
}

\author{
Norazlina Subani, Norsarahaida Amin \\ Department of Mathematical Sciences, Faculty of Science, Universiti Teknologi Malaysia, 81310 UTM Johor Bahru, Johor, Malaysia \\ * Corresponding author: norsarahaida@utm.my
}

\section{Article history}

Received 19 October 2017

Accepted 8 November 2017

\begin{abstract}
This work focuses on the development of a mathematical model as a viable alternative to pinpoint locations of gas leaks in a pipeline. The transient non-isothermal flow of hydrogen-natural gas mixture is considered because hydrogen is often transported in the same pipeline as natural gas to reduce the transportation cost. The mathematical model developed took into consideration the effect of the mass ratio of gas mixture. The gas mixture was assumed to be homogeneous and the transient pressure wave was created by the sudden or instantaneous closure of a downstream shutoff valve to ensure the attainment of minimum pressure at the downstream end within a short time. The governing equations were numerically solved using the reduced order modelling (ROM) technique, which had not been previously applied on non-isothermal models involving gas mixtures. Numerical results observed that the mass ratio of hydrogen to natural gas should not be more than 0.5 to ensure that leakage does not occur before the estimated leak position. An increase in the mass ratio $\phi$ leads to an increase in the pressure and celerity wave, while the leak location and the amount of leak discharge decrease.
\end{abstract}

Keywords: Leak detection, gas mixture, hydrogen, natural gas, reduced order modelling

\section{INTRODUCTION}

Hydrogen could be considered as a renewable energy and a fuel alternative in our future energy demand and it is the most abundant and cleanest gas (Corbo et al., 2011). It is colourless, odourless, tasteless and non-toxic, but highly flammable at atmospheric pressure and temperature (Cheng et al., 2009; Tabkhi et al., 2008). Hydrogen could play an important role as a sustainable energy supply, since the utilization of hydrogen yields no carbon dioxide $\left(\mathrm{CO}_{2}\right)$. If hydrogen is made from renewable energy sources without yielding much $\mathrm{CO}_{2}$, it would be possible to produce and use energy with near zero emissions of greenhouse gases or air pollutants (Chiesa et al., 2001).

Although hydrogen could be considered as the alternative fuel, it has a disadvantage in terms of storage and backfire phenomenon (Sierens and Rosseel, 2000). It does not exist on its own and has a storage problem. It is not easy to store hydrogen gas or transport by vehicle because of its low density and temperature. Because of its low volumetric energy content, an estimated pressure at above 200 bars is needed to transport the hydrogen over long distances (Elaoud and Hadj-Taïeb, 2008). Due to the lower heating value, hydrogen pipelines need to operate at higher pressures to achieve comparable energy transport capacities or they have to be of large diameter (Weber and Perrin, 2008). High pressure gas requires steel pipes for effective transportation.

Significant efforts have been made in many researches towards the reduction of hydrogen production cost. In an effort made by various stakeholders in need of using hydrogen as fuel to replace fossil fuels, researches have been conducted on the possibility of mixing hydrogen with natural gas as a starting point towards a full fledge hydrogen usage (Hoeseldonackx and D'haeseleer, 2011). Thus, hydrogen has been found to occur chiefly in combination with other gases such as natural gas. Some percentage of hydrogen can be added to natural gas design engines which will improve its performance (Bade and Karim, 1999; Veziroglu and Barbir, 1992). A combination of hydrogen and natural gas can also serve as a testing ground towards free emission energy source since hydrogen is a nonemission fuel.

Distribution of hydrogen in pipelines actually is the most costeffective way to supply large amounts of gas. Pipeline transportation of hydrogen and natural gas remains the cheapest and safest means of transportation (Elaoud and Hadj-Taïeb, 2008; Elaoud et al., 2014; Peet et al., 2009; Weber and Perrin, 2008). This is expected to reduce with the introduction of hydrogen energy. Mixing hydrogen with natural gas can improve the storage capability and at the same time, enhance the low burning capacity of natural gas. However, mixing higher percentages of hydrogen $(<40 \%)$ requires special attention regarding the functioning of pipeline, end-user appliances and emissions (Uilhoorn, 2009). In the gas pipeline transportation system, the existing pipe is designed and constructed specifically for one gas only, most are natural gas. Hydrogen is a reactive element and it diffuses into the materials such as the steel pipelines and this could cause changes in the mechanical properties and could lead to rupture or leakages.

According to Subani et al. (2017), pipeline companies are facing a major challenge to detect and locate leakages. From the 3751 pipe incidents that were recorded between 1994 and 1999, two third of 
them were as a result of pipe leaks (Lydell, 2000). Leakage in pipelines can cause serious problems related not only to the environment or safety, but also to the economy (Elaoud et al., 2010). Leaks also waste natural resources and create public health risks. Existing studies mainly use sensors to detect and determine the onset of leakage, but these sensors, depending on their types are expensive to install. They could also give rise to false alarms and their handling needs skilled operators. As such, mathematical modelling has been adopted to be a viable alternative that is highly sensitive to pinpoint the leak location even for small leaks and to minimize the occurrence of false alarms at low cost.

However, risk of leakage through pipelines is well studied for natural gas (Turner and Mudford, 1988; Wilkening and Baraldi, 2007), but not for hydrogen or hydrogen-natural gas mixtures. The mixture needs to be considered because hydrogen is often added to natural gas to enhance the latter's burning capacity, and because hydrogen needs to be transported in the same pipeline as natural gas due to its storage problem and to reduce transportation cost. Agaie et al. (2017), Elaoud and Hadj-Taïeb (2009), Elaoud et al. (2010) and Subani et al. (2015) determined the leakage of hydrogen-natural gas mixtures in pipelines, but they assumed the transient isothermal flow. They leave out the gravity and inclination terms in the momentum equation, and they do not consider the energy equation in their models.

The main objective of this paper is to determine and locate leakage on non-isothermal transient flow in the inclined pipeline system of hydrogen-natural gas mixtures based on transient pressure wave analysis. The effect of the hydrogen mass ratio of the transient flow of hydrogen-natural gas mixture is analyzed. The gas mixture was assumed to be homogeneous and the transient pressure wave was created by the sudden or instantaneous closure of a downstream shutoff valve to ensure the attainment of minimum pressure at the downstream end within a short time. The governing equations are numerically solved using the reduced order modelling (ROM) technique, which had not been previously applied on non-isothermal models involving gas mixtures (Agaie and Amin, 2014; Agaie et al., 2017; Behbahani-Nejad and Shekari, 2008; 2010; Subani and Amin, 2015; Subani et al., 2015).

\section{MATHEMATICAL FORMULATION}

The governing equations consist of three coupled non-linear hyperbolic partial differential equations. The flow is assumed to be one dimensional, non-isothermal, compressible and include transient condition. The fluid is assumed to be a homogeneous mixture of hydrogen and natural gas where both fluids are mixed together in one layer and no inter-phase interaction between them.

\section{Governing equation}

From the principle of conservation of mass, momentum and energy laws, the governing equations for the transportation of hydrogen-natural gas mixtures in an inclined pipeline are given by the following:

$$
\begin{aligned}
& \frac{\partial \rho}{\partial t}+\frac{\partial(\rho u)}{\partial x}=0 \\
& \frac{\partial(\rho u)}{\partial t}+\frac{\partial\left(\rho u^{2}+P\right)}{\partial x}+\frac{f \rho u|u|}{2 D}+\rho g \sin \theta=0 \\
& \frac{\partial(\rho e)}{\partial t}+\frac{\partial(\rho u e+P u)}{\partial x}=\rho q-\rho u g \sin \theta
\end{aligned}
$$

where $\rho$ is defined as density, $u$ is the gas velocity where the modulus sign is to ensure that the frictional force shall always act opposite to the direction of motion, $P$ is the pressure, $f$ is the coefficient of friction, $D$ is the diameter of the pipeline, $g$ is the gravitational force, $\theta$ is an angle between the friction force and the $x$ direction, $e$ is the internal energy and $q$ is heat transfer.
These governing equations (1), (2) and (3) will be used in transient analysis of non-isothermal hydrogen-natural gas mixture in the pipeline where kinetic energy $u^{2} / 2$ is neglected, as shown in Fig. 1 .

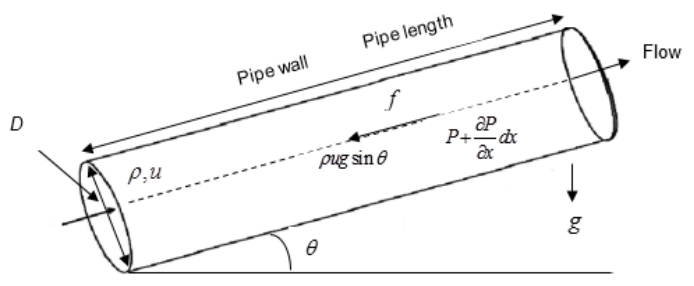

Fig. 1 General flow of gas pipeline.

\section{Equation of state}

An equation of state for a gas relates the variables of pressure, density and temperature. The equation of state for perfect gas, which is commonly used in the gas industry, is given by:

$$
P=\rho R T
$$

where $R$ is the specific gas constant and $T$ is the temperature. For compressible flow, the relation of equation of state with the celerity pressure wave $c$ is denoted as:

$$
P=\rho c^{2}
$$

From the relation of a perfect gas, the specific heat at constant volume $C_{v}$ and internal energy $e$ are defined as:

$$
C_{v}=\frac{R}{r-1} \quad \text { and } \quad e=C_{v} T
$$

where $r$ is constant. Substituting equation (6) into the equation (4), then the perfect gas equation is written as:

$$
P=\rho C_{v}(r-1) \frac{e}{C_{v}}=(r-1) \rho e
$$

\section{Hydrogen-natural gas mixture equation}

Density of hydrogen and natural gas is defined as:

$$
\rho_{h}=\frac{m_{h}}{V_{h}} \quad \text { and } \quad \rho_{g}=\frac{m_{g}}{V_{g}}
$$

where $m_{g}, m_{h}, V_{g}$ and $V_{h}$ are defined as the mass of natural gas and hydrogen and volume of natural gas and hydrogen, respectively.

For hydrogen-natural gas mixtures, the hydrogen mass ratio will be used in determining the mixture density, where the mass ratio of the mixture is given as:

$$
\phi=\frac{m_{h}}{m_{h}+m_{g}}
$$

From the definition of density of gas, the density of hydrogennatural gas mixture is defined as:

$$
\frac{1}{\rho}=\frac{V_{m}}{M_{m}}
$$

where $V_{m}=V_{h}+V_{g}$ and $M_{m}=m_{h}+m_{g} . V_{m}$ and $M_{m}$ are defined as the volume and mass of the hydrogen-natural gas mixture. Substituting equations (8) and (9) into the equation (10), the density of 
hydrogen-natural gas mixture is defined according to the mass ratio $\phi$, given by:

$$
\frac{1}{\rho}=\frac{m_{h}}{\rho_{h}\left(m_{h}+m_{g}\right)}+\frac{m_{g}}{\rho_{g}\left(m_{h}+m_{g}\right)}
$$

and

$$
\rho=\left[\frac{\phi}{\rho_{h}}+\frac{(1-\phi)}{\rho_{g}}\right]^{-1}
$$

The density of hydrogen and natural gas, according to isentropic laws is written as:

$$
\rho_{h}=\rho_{h_{0}}\left(\frac{P}{P_{0}}\right)^{\frac{1}{n^{\prime}}} \quad \text { and } \quad \rho_{g}=\rho_{g_{0}}\left(\frac{P}{P_{0}}\right)^{\frac{1}{n^{\prime \prime}}}
$$

where each $\rho_{h_{0}}$ and $\rho_{g_{0}}$ represents the initial density of hydrogen and natural gas, respectively. $P$ is the transient pressure and $P_{0}$ is permanent pressure, $n^{\prime}$ and $n^{\prime \prime}$ represent the flow process index of hydrogen and natural gas, respectively.

To solve the governing equations (1), (2) and (3) numerically, the density of the hydrogen-natural gas mixture $\rho$ must be expressed according to the gas pressure $P$. Substituting equation (12) into equation (11), the expression of the average density of the mixture is given by:

$$
\rho=\left[\frac{\phi}{\rho_{h_{0}}}\left(\frac{P_{0}}{P}\right)^{\frac{1}{n^{\prime}}}+\frac{(1-\phi)}{\rho_{g_{0}}}\left(\frac{P_{0}}{P}\right)^{\frac{1}{n^{\prime \prime}}}\right]^{-1}
$$

For compressible flow, the celerity of the pressure wave is defined as:

$$
c^{2}=\left(\frac{\partial P}{\partial \rho}\right)_{s} \quad \text { thus } \quad c=\left(\frac{\partial \rho}{\partial P}\right)_{s}^{-\frac{1}{2}}
$$

where the subscript $s$ denotes the condition of constant entropy. By taking the derivative of equation (13) with respect to $P$, and substituting into equation (14), then the celerity pressure wave yields:

$$
\begin{aligned}
c= & {\left[\frac{\phi}{\rho_{h_{0}}}\left(\frac{P_{0}}{P}\right)^{\frac{1}{n^{\prime}}}+\frac{(1-\phi)}{\rho_{g_{0}}}\left(\frac{P_{0}}{P}\right)^{\frac{1}{n^{\prime \prime}}}\right] \times } \\
& {\left[\frac{1}{P}\left\{\frac{\phi}{n^{\prime} \rho_{h_{0}}}\left(\frac{P_{0}}{P}\right)^{\frac{1}{n^{\prime}}}+\frac{(1-\phi)}{n^{\prime \prime} \rho_{g_{0}}}\left(\frac{P_{0}}{P}\right)^{\frac{1}{n^{\prime \prime}}}\right\}\right]^{-\frac{1}{2}} }
\end{aligned}
$$

\section{Boundary conditions}

The boundary conditions depend on the valve operational time and the types of closure. The boundary conditions at the initial point, $x=0$, are given by:

$$
\begin{aligned}
& \rho(0, t)=\rho_{0}(t) \\
& \rho u(0, t)=m_{0}(t) \\
& T(0, t)=T_{0}(t)
\end{aligned}
$$

where $\rho_{0}, m_{0}$ and $T_{0}$ are defined as density, mass flux and temperature at the inlet pipeline, respectively.
The boundary conditions at the end point $x=L$ are:

$$
\begin{aligned}
& \rho(L, t)=\rho_{L}(t) \\
& \rho u(L, t)=m_{L}(t) \\
& \rho u e(L, t)=h_{L}(t)
\end{aligned}
$$

where $\rho_{L}, m_{L}$ and $h_{L}$ are defined as density, mass flux and heat flux at the outlet pipeline, respectively.

\section{Initial conditions}

The initial conditions that are assumed to be in a steady state condition at $t=0$ are:

$$
\begin{aligned}
& \frac{\partial \rho u}{\partial x}(x, 0)=0 \\
& \frac{\partial \rho u^{2}}{\partial x}(x, 0)=-c^{2} \frac{\partial \rho}{\partial x}-\frac{f \rho u|u|}{2 D}-\rho g \sin \theta \\
& \frac{\partial(\rho u e)}{\partial x}(x, 0)=-\frac{\partial P u}{\partial x}+\rho q-\rho u g \sin \theta
\end{aligned}
$$

\section{NUMERICAL SOLUTION}

To solve the governing equations (1), (2) and (3) numerically, the ROM is employed (Agaie, 2014; Agaie and Amin, 2014; BehbahaniNejad and Shekari, 2008; 2010; Behbahani-Nejad et al., 2004; Subani and Amin, 2015; Subani et al., 2015; 2017). These governing equations are transformed in the flux vector form as:

$$
\frac{\partial Q}{\partial t}+\frac{\partial E(Q)}{\partial x}-H(Q)=0
$$

where

$$
Q=\left[\begin{array}{c}
\rho \\
\rho u \\
\rho e
\end{array}\right], E(Q)=\left[\begin{array}{c}
\rho u \\
\rho u^{2}+P \\
\rho u e+P u
\end{array}\right], H(Q)=\left[\begin{array}{c}
0 \\
-\frac{f \rho u|u|}{2 D}-\rho g \sin \theta \\
\rho q-\rho u g \sin \theta
\end{array}\right] .
$$

\section{Implicit Steger-Warming flux vector splitting scheme}

The implicit Steger-Warming flux vector splitting method (FSM) is used as the numerical scheme (Hoffmann and Chiang, 2000; Subani et al., 2015; 2017). The governing equation (19) is discretized using FSM, and the resulting FSM scheme in the delta notation is:

$$
\begin{aligned}
& {\left[\frac{\Delta t}{\Delta x} A_{M i+1}^{n(-)}\right] \Delta Q_{i+1}+\left[I+\frac{\Delta t}{\Delta x}\left(A_{M i}^{n(+)}-A_{M i}^{n(-)}\right)-\Delta t B_{M i}{ }^{n}\right] \Delta Q_{i}-} \\
& {\left[\frac{\Delta t}{\Delta x} A_{M i-1}^{n(+)}\right] \Delta Q_{i-1}=-\frac{\Delta t}{\Delta x}\left[E_{i}^{n(+)}-E_{i-1}^{n(+)}+E_{i+1}^{n(-)}-E_{i}^{n(-)}\right]+} \\
& \Delta t H_{i}^{n}
\end{aligned}
$$

where

$$
\Delta Q=Q^{n+1}-Q^{n}
$$

and $I$ is the identity matrix, and $A_{M}$ and $B_{M}$ are the Jacobian matrices defined as:

$$
A_{M}=\frac{\partial E(Q)}{\partial Q} \quad \text { and } \quad B_{M}=\frac{\partial H(Q)}{\partial Q}
$$

Since the flux vector $E$ possesses the homogeneous property, therefore, it may be split into sub-vectors such that each sub-vector is associated with a positive and negative eigenvalues of the flux matrix 
Jacobian $A_{M}=A_{M}^{+}+A_{M}^{-}$. Noted that the governing equation has the property that the flux vector $E$ is a homogeneous function of degree one in $Q$. Therefore, the flux vector $E$ is defined as $E=A_{M} Q$ and $A_{M}$ exist two sub vector associated positive and negative parts for homogeneous $E(Q)$, where $E$ has positive and negative terms defined as $E=E^{+}+E^{-}$(Hoffmann and Chiang, 2000). From equation (20), the Jacobian matrix $A_{M}$ is split into two parts (positive and negative) as denoted by $A_{M}{ }^{+}$and $A_{M}{ }^{-}$:

$$
\begin{gathered}
A_{M}{ }^{+}=\frac{u}{2 c}\left[\begin{array}{ccc}
-u+c & \frac{u+c}{u} & \frac{(r-1)(c-u)}{c u} \\
-\left(u^{2}+c^{2}\right) & \frac{(u+c)^{2}}{u} & \frac{(r-1)(u+c)}{u} \\
-\frac{r c^{2}(u+c)}{r-1} & \frac{r c^{2}(u+c)}{u(r-1)} & \frac{r c(u+c)}{u}
\end{array}\right] \\
A_{M}{ }^{-}=\frac{u}{2 c}\left[\begin{array}{ccc}
u-c & \frac{c-u}{u} & \frac{(r-1)(u-c)}{c u} \\
(u-c)^{2} & -\frac{(u-c)^{2}}{u} & \frac{(r-1)(c-u)}{u} \\
\frac{r c^{2}(u-c)}{r-1} & -\frac{r c^{2}(u-c)}{u(r-1)} & \frac{r c(u-c)}{u}
\end{array}\right]
\end{gathered}
$$

while $E^{+}$and $E^{-}$are the positive and negative parts of the flux vector $E$ given by:

$$
E^{+}=\left[\begin{array}{c}
\frac{\rho(u+c)}{2} \\
\frac{\rho(u+c)^{2}}{2} \\
\frac{r \rho c^{2}(u+c)}{2(r-1)}
\end{array}\right] \text { and } E^{-}=\left[\begin{array}{c}
\frac{\rho(u-c)}{2} \\
\frac{\rho(u-c)^{2}}{2} \\
\frac{r \rho c^{2}(u-c)}{2(r-1)}
\end{array}\right]
$$

When equation (20) is applied to each grid point, a block tridiagonal of systems of algebraic equations is obtained. The system of equations is solved at each time step and results in $\Delta Q$. The values of $Q$ at the next time step are calculated using the equation (21). To perform the Eigen analysis and to construct ROM technique based on the flow eigenmodes, the finite difference equation in the equation (20) is linearized. The linearization can be achieved by assuming steady state results in which stability perturbation is used to obtain the transient solution at each nodal point. For linearization, the flow field variables at each time step are considered as:

$$
Q^{n+1}=Q^{0}+\hat{Q}^{n+1}
$$

where $Q^{n+1}=Q^{0}+\hat{Q}^{n+1}$ represents the corresponding steady state values, and $\hat{Q}$ represents the perturbation values. Substituting equation (25) into equation (20) yields:

$$
\begin{aligned}
& -\left[\frac{\Delta t}{\Delta x} A_{M i-1}^{0(+)}\right] \hat{Q}_{i-1}^{n+1}+\left[I+\frac{\Delta t}{\Delta x}\left(A_{M i}^{0(+)}-A_{M i}^{0(-)}\right)\right] \hat{Q}_{i}^{n+1} \\
& +\left[\frac{\Delta t}{\Delta x} A_{M i+1}^{0(-)}\right] \hat{Q}_{i+1}^{n+1}-\Delta t B_{M i}^{0} \hat{Q}_{i}^{n+1}=\hat{Q}_{i}^{n}
\end{aligned}
$$

The above linearization of equation (26) is represented in the form of the following eigenvalue problem:

$$
W^{0} \hat{Q}^{n+1}=I \hat{Q}^{n}+V^{n+1}
$$

where $V$ is defined as a vector consisting the known values by the boundary conditions $\quad V^{n+1}=-\left[\frac{\Delta t}{\Delta x} A_{M i-1}^{0(+)}\right] \hat{Q}_{i-1}^{n+1}+\left[\frac{\Delta t}{\Delta x} A_{M i+1}^{0(-)}\right] \hat{Q}_{i+1}^{n+1}$, and $W^{0}$ represents the coefficient of the matrix made by the left hand side terms of equation (27) $W^{0}=\left[I+\frac{\Delta t}{\Delta x} A_{M i}{ }^{0(+)}-A_{M i}{ }^{0(-)}\right]-\Delta t B_{M i}^{0}$. The finite difference equation (26) is the linearized form of FSM.

\section{Reduced order modelling}

To construct the ROM technique, the zero eigenvalue in the Eigen system of matrix is required. For zero forcing function $V$, the homogeneous part of the equation (27) needs to be considered by setting:

$$
\hat{Q}_{i}=x_{i} \exp \left(\lambda_{i} t\right) \quad \text { and } \quad z_{i}=\exp \left(\lambda_{i} \Delta t\right)
$$

where $\lambda_{i}$ and $z_{i}$ are defined as $i$-th eigenvalues, and $x_{i}$ is eigenvector (Giles, 1983; Hall, 1994; Michael and Earl, 1996).

Equation (28) is used to obtain the following generalized eigenvalue problem. Then, the general form of the diagonal matrix which contains the eigenvalues and eigenvectors are written as:

$$
Z W^{0} X=I X
$$

where $Z$ represents the diagonal matrix of eigenvalues at each time step, and $X$ is the matrix with columns that are the right eigenvector.

On the other hand, considering the left eigenvectors of the homogeneous part of the equation (27) $W^{0} \hat{Q}^{n+1}=I \hat{Q}^{n}$ satisfy the following relation:

$$
\left(W^{0}\right)^{T} Y Z=I Y
$$

where $Y$ is the matrix with rows that are the left eigenvectors. The eigenvectors are then normalized to satisfy the following orthogonality conditions:

$$
Y^{T} W^{0} X=I \quad \text { and } \quad Y^{T} I X=Z
$$

Agaie (2014), Agaie and Amin (2014) and Subani et al. (2015) analyzed the eigenmodes based on the time basis, then the equation (28) reduces the gas flow behaviour to the sum of individual nodes:

$$
\hat{Q}=X \hat{c}
$$

where $\hat{Q}$ and $\hat{c}$ are defined as the vector of normal node coordinates and denoted by:

$$
\hat{Q}=\left[\begin{array}{c}
Q_{1} \\
Q_{2} \\
\mathrm{M} \\
Q_{n}
\end{array}\right] \quad \text { and } \quad \hat{c}=\left[\begin{array}{c}
\hat{c}_{1} \\
\hat{c}_{2} \\
\mathrm{M} \\
\hat{c}_{n}
\end{array}\right]
$$

Multiplying equation (32) by $Y^{T} W^{0}$ we have:

$$
\hat{c}=Y^{T} W^{0} Q
$$

Substituting equations (31) and (27) and then multiplying by $Y^{T}$ giving a set of $N$ uncoupled equations for the nodal coordinates $\hat{c}$ :

$$
\hat{c}^{n+1}=Z \hat{c}^{n}+Y^{T} V^{n+1}
$$

Since the orthogonality conditions are satisfied, the eigenmodes can be retained to construct ROM by using equation (35). The results are reliable and steady if the zero and negative eigenvalues are 
removed. Then, the transient flow of hydrogen-natural gas mixture now is analyzed using equation (35) which contains the results of nonzero and positive eigenvalues.

\section{Treatment of boundary conditions}

The boundary conditions have been developed for the governing equations (1), (2) and (3). The finite difference equations developed for the treatment of boundary conditions are given as:

$$
\begin{aligned}
\rho_{0}^{n+1}= & \rho_{0}^{n}+\frac{\Delta t}{\Delta x}\left(m_{0}^{n}-m_{1}^{n}\right) \\
m_{0}^{n+1}= & m_{0}^{n}-\frac{\Delta t}{\Delta x}\left[\left(\frac{m_{1}^{n^{2}}}{\rho_{1}^{n}}+c_{1}^{n^{2}} \rho_{1}^{n}\right)-\left(\frac{m_{0}^{n^{2}}}{\rho_{0}^{n}}+c_{0}^{n^{2}} \rho_{0}^{n}\right)\right]- \\
& \frac{f}{2 D} \frac{m_{0}^{n}\left|m_{0}^{n}\right|}{\rho_{0}^{n}} \Delta t-\rho_{0}^{n} g \sin \theta \\
h_{0}^{n+1}= & h_{0}^{n}-\frac{\Delta t}{\Delta x}\left[\left(\frac{h_{1}^{n} m_{1}^{n}}{\rho_{1}^{n}}+c_{1}^{n^{2}} m_{1}^{n}\right)-\left(\frac{h_{0}^{n} m_{0}^{n}}{\rho_{0}^{n}}+c_{0}^{n^{2}} m_{0}^{n}\right)\right]+ \\
& \rho_{0}^{n} q+m_{0}^{n} g \sin \theta
\end{aligned}
$$

where $m_{0}$ and $m_{1}$ are the mass flux before and after leak point, $\rho_{0}$ and $\rho_{1}$ are density of gas mixture before and after leak point, $c_{0}$ and $c_{1}$ are celerity wave of gas mixture before and after leak point, and $h_{0}$ and $h_{1}$ are the heat flux of gas mixture before and after leak point, respectively.

\section{LEAK DETECTION}

The problem is focused on transients occurring in a single inclined pipeline with a compressor at the upstream end and a sudden closure valve at the downstream end as illustrated in Fig. 2. A single leak is supposed to exist at an intermediate section of the pipeline and located at a distance $X_{L}$ from the compressor. The pipeline with a length $L$ is divided into two segments, which are Pipe 1, from the compressor to the leak, and Pipe 2 from the leak to the valve.

\section{Leak modelling}

The leak orifice can be simulated based on the flow rate through a small orifice between the high-pressure pipeline and the environment. The discharged flow $Q_{1}$ from the orifice can be calculated by the given equation:

$$
Q_{1}=\left(\rho_{1} C_{d} A_{1} \sqrt{2 P_{1} / \rho_{1}}\right) / X_{L}
$$

where $C_{d}$ is a discharge coefficient, $A_{1}$ is the orifice area of the leak with radius $r_{1}, P_{1}$ and $\rho_{1}$ are the pressure and the density of gas mixture at the leak position, respectively and $X_{L}$ is the distance of leak from the reservoir.

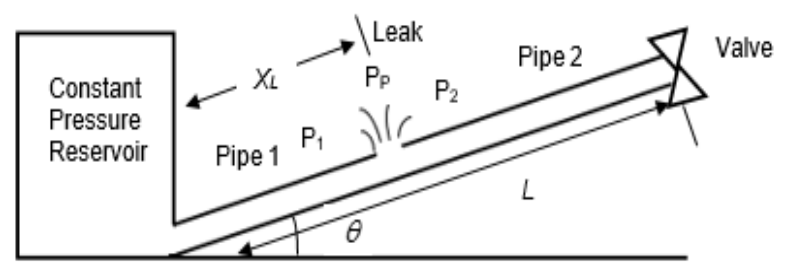

Fig. 2 Hydrogen-natural gas mixture installation with leakage in an inclined pipeline.
The analysis of the transient pressure wave of hydrogen-natural gas mixtures are based on the properties of transmission and reflection of pressure wave caused by a sudden closure of a downstream valve. When the initial pressure wave reaches the leak, it will produce a reflection when it arrives back at the downstream end section. The time difference between the initial transient wave and the reflected wave is then measured, and the position of leakage $X_{L}$ in the pipeline is calculated by:

$$
X_{L}=L-\frac{\Delta t_{l} c}{2}
$$

where $X_{L}$ is the distance to the leak from the upstream end section, $\Delta t_{l}$ is the time difference between the initial transient wave and the reflected wave and $c$ is the transient celerity wave.

By considering the partial reflection of pressure waves which take place at the leak, the discharged behaviour of the leak may be obtained by:

$$
Q_{L}=\frac{A}{c}\left(P_{0}^{*}-P_{1}^{*}\right)
$$

where $Q_{L}$ is the leak discharge for transient flow, $A$ is an area of pipeline at leak point, $P_{0}^{*}$ and $P_{1}^{*}$ are the initial pressure rises produced by the sudden closure of the downstream valve and the pressure at leak point, respectively.

\section{Boundary conditions when a leak occurs}

The transient flow is created by the sudden closure of the valve at the downstream end $(x=L)$. At this point the discharge flow of leakage is $Q_{2}(L, t)=0$. At the upstream end ( $x=0$ and $\left.t>0\right)$, the condition of the reservoir is given by the constant pressure $P_{1}(0, t)=P_{0}$. At the leak point, the equation (37) is implemented as the internal boundary condition. According to Elaoud et al. (2010), the two relationships that relate the upstream pressure and discharge flow to the downstream pressure and discharge flow are:

$$
\begin{aligned}
& P_{1}=P_{2}=P_{p} \\
& Q_{1}=Q_{2}+\left(\rho_{P} C_{d} A_{1} \sqrt{2 P_{P} / \rho_{P}}\right) / X_{L}
\end{aligned}
$$

where $P_{1}, P_{2}$ and $P_{p}$ are the pressure before, after and at leak point, respectively (refer to Fig. 2). $Q_{1}$ and $Q_{2}$ are the discharge flow at upstream and downstream of the leak, respectively, and $\left(\rho_{P} C_{d} A_{1} \sqrt{2 P_{P} / \rho_{P}}\right) / X_{L}$ is the leakage mass flow rate.

\section{RESULTS AND DISCUSSION}

The proposed numerical method ROM technique is validated with Elaoud et al. (2010) by comparing the present numerical method with previous numerical on hydrogen-natural gas mixture with mass ratio $\phi=0, \phi=0.5$ and $\phi=1$ at horizontal pipeline. From the comparison, a good agreement was observed on the validated results. In the present study, the ROM technique is used to analyze the flow characteristics of hydrogen-natural gas mixture in an inclined pipeline $\theta=15^{\circ}$.

The effects of the mass ratio of gas mixture on the flow characteristics of hydrogen-natural gas mixture in an inclined pipeline are investigated. The computational domain is discretized into 1000 segments. The domain is divided into 1000 segments to acquire better results. An increase in the number of segments in an increases the accuracy of the results, but does not affect the computation time. To obtain the ROM method, the FSM is used as numerical scheme. From this scheme, the results can be directly achieved, but it takes long time 
to compute the results. Thus, this present method ROM is proposed to reduce the computational time during the analysis.

\section{Comparison between current method and existing method on pressure distribution}

The results are presented and discussed to validate the present numerical method ROM with the existing numerical method. The results on pressure distribution are compared with the results on Elaoud et al. (2010), where the previous study has considered a characteristics method. The mathematical model considers the flow is compressible and potential energy may be ignored. The transient flow is supposed to be one dimensional and the gas mixture of hydrogennatural gas is assumed to be homogeneous. The governing equations (1) and (2) with the boundary and initial conditions $(16 a, b),(17 a, b)$ and $(18 \mathrm{a}, \mathrm{b})$ are considered.

A single horizontal pipeline with a constant pressure reservoir at the upstream end and a linear closing valve at the downstream end are considered. A single leak is supposed to exist at an intermediate section of the pipe and located at a distance $X_{L}$ from the reservoir. The pipe is placed horizontally or lie at the same height, $\theta=0^{\circ}$. The pipe with a length $L$ is subdivided into two segments, which is Pipe 1 , from the reservoir to the leak, and Pipe 2 from the leak to the valve as illustrated in Fig. 3.

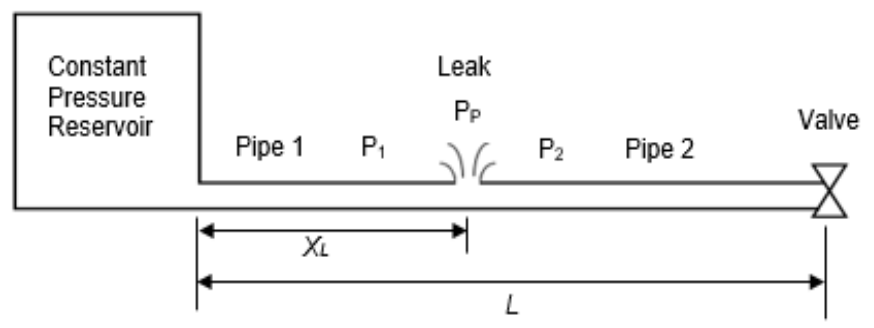

Fig. 3 Hydrogen-natural gas mixture installation with leakage in a rigid horizontal pipeline (Elaoud et al., 2010).

\section{Description of problem}

This work focused on the development of a mathematical model to detect and locate leaks for transient non-isothermal flow of hydrogen-natural gas mixture in an inclined pipeline. To locate the leak by the transient flow analysis of hydrogen-natural gas mixtures, an iron pipe $0.4 \mathrm{~m}$ in diameter and $600 \mathrm{~m}$ long are considered. The mixture is considered as hydrogen is often added to natural gas to enhance the latter's burning capacity, and because hydrogen needs to be transported in the same pipeline as natural gas due to its storage problem and to reduce transportation cost. The gas mixture was assumed to be homogeneous. The pipe is placed at different angles ( $\left.\theta=0^{\circ}, \theta=15^{\circ}\right)$ and connected to a constant pressure reservoir at the upstream end, while the sudden/instantaneous closure valve is located at the downstream end to ensure the attainment of minimum pressure within a short time as indicated in Fig. 2.

The boundary conditions imposed were under the assumption that a reservoir exists at the upstream and a sudden closure valve was at the downstream. The governing equations (1), (2) and (3) with the boundary and initial conditions (16a, b, c), (17a, b, c) and (18a, b, c) are considered and were numerically solved using the reduced order modelling (ROM) technique. A mass flow $Q_{0}=55 \mathrm{~kg} / \mathrm{s}$ at a static temperature $T=15^{\circ} \mathrm{C}$ and an absolute pressure $P=35$ bar are assumed. The properties of hydrogen and natural gas used in the calculations are those of Elaoud et al. (2010) and Subani et al. (2015).

\section{The effect of mass ratio of gas mixture}

Fig. 5 shows the plots of the transient pressure as a function of time with a sudden closure valve at leakage $X_{L}=L / 3$ for different mass ratio of hydrogen-natural gas mixtures $\phi$ at horizontal pipeline and different angles $\theta=15^{\circ}$. As indicated in Fig. 5, the transient pressure waves are dependent on inclination angle $\theta$ and the mass ratio of gas mixture $\phi$. Based on this Fig. 5, an increase in the angles of pipeline $\theta$ and the mass ratio of gas mixture $\phi$ results in an increase in the transient pressure distribution. As presented in Fig. 5, the leak point is occurring at $t=0.81 \mathrm{~s}$. It is also observed that an increase in the inclination angle $\theta$ and the mass ratio of gas mixture $\phi$ leads to an increase in the pressure drop. This is because hydrogen $(\phi=1)$ has higher pressure than natural gas $(\phi=0)$.

For horizontal pipeline $\theta=0^{\circ}$, the pressure of hydrogen-natural gas mixture at leak point $200 \mathrm{~m}$ for mass ratio of hydrogen to natural gas at natural gas at $\phi=0, \phi=0.25, \phi=0.5, \phi=0.75$ and $\phi=1$ are 27.40 bar, 27.80 bar, 28.02 bar, , 28.04 bar and 28.06 bar, respectively. When the angle increase at $\theta=15^{\circ}$, the pressure of hydrogen-natural gas mixture at $\phi=0, \phi=0.25, \phi=0.5, \phi=0.75$ and $\phi=1$ are decreased to $27.31 \mathrm{bar}, 27.40 \mathrm{bar}, 27.60 \mathrm{bar}$, 27.72 bar and 27.84 bar, respectively. Noted that, an increase in the mass ratio $\phi$ lead to a decrease the pressure dropped, while an increase in the inclination angles $\theta$ lead to an increase in the pressure dropped.

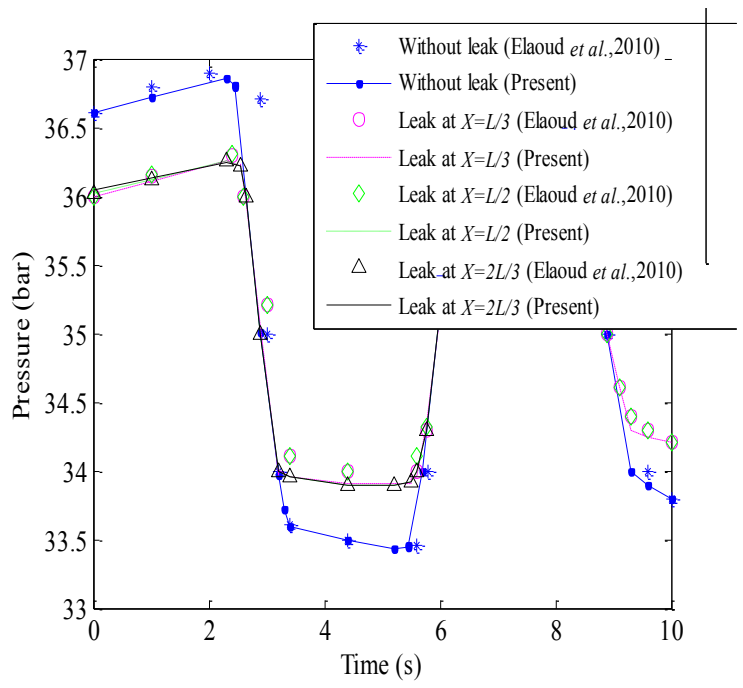

(a) Natural gas $(\phi=0)$

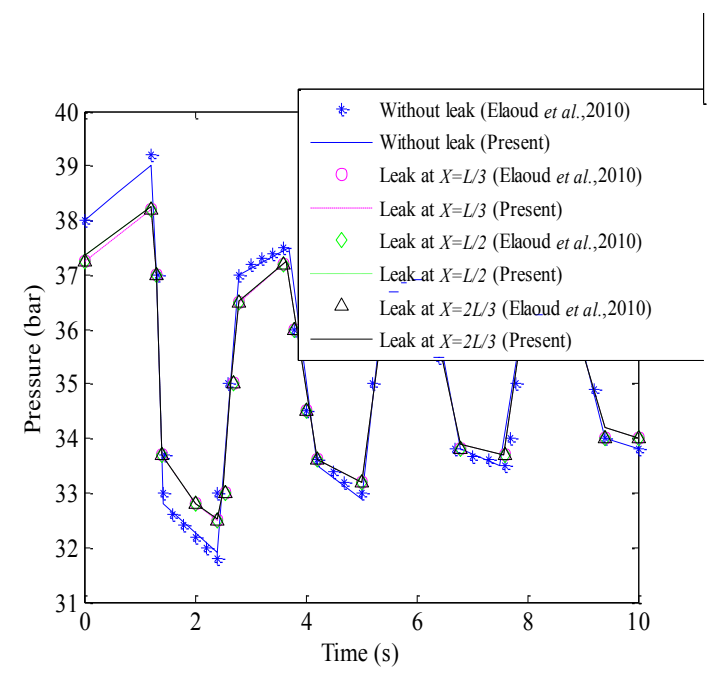

(b) Gas mixture $(\phi=0.5)$ 


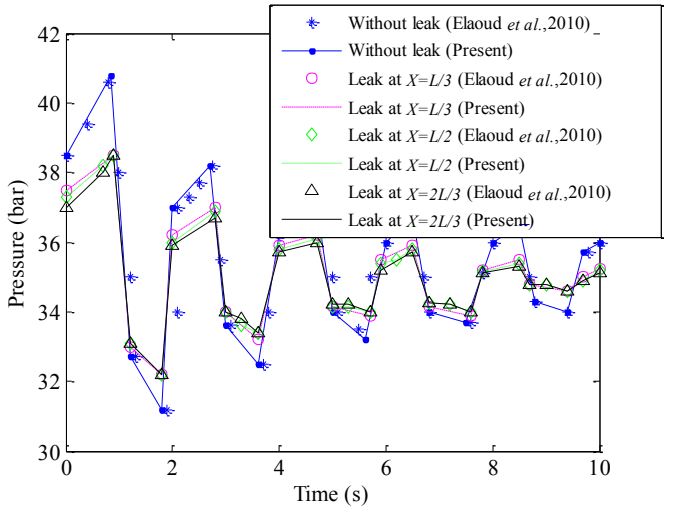

(c) Hydrogen $(\phi=1)$

Fig. 4 Transient pressure distribution for the linear closure valve at the downstream end of the pipe for different values of mass ratio $\phi$ (Elaoud et al., 2010) validated with ROM method.

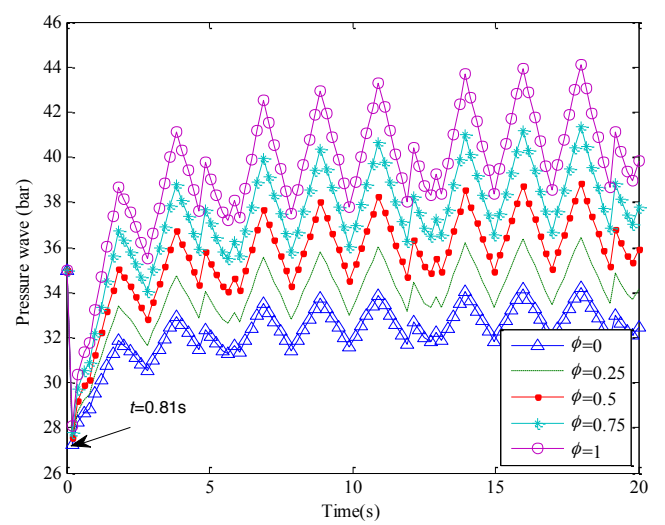

(a) Horizontal pipeline $\theta=0^{\circ}$

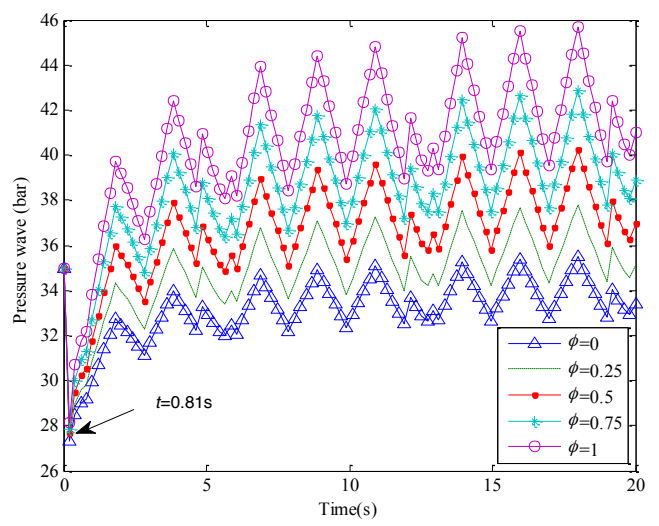

(b) Inclined pipeline $\theta=15^{\circ}$

Fig. 5 Transient pressure distribution of hydrogen-natural gas mixture $\phi=0, \phi=0.25, \phi=0.5, \phi=0.75$ and $\phi=1$.

Based on Fig. 5, the pressure dropped at $414.1 \mathrm{~ms}^{-1}$, represented the leak occurs at this time. At this point, the position of leakage and the amount of leak discharge will be determined. After the leak point $t>0.81 \mathrm{~s}$, the pressure increased and decreased, then starting to oscillate until the maximum time $t=20.0 \mathrm{~s}$. Means that the pressure starts to reduce after the pressure reached to maximum point and continue oscillating until the valve is fully closed at the downstream end of the pipeline. After $t=20.0 \mathrm{~s}$, the pressure has not exceed to the maximum point because the pressure starts to reduce at $t=19.0 \mathrm{~s}$ and remain reduced after that for each mass ratio ( $\phi=0, \phi=0.25$, $\phi=0.5, \phi=0.75$ and $\phi=1)$ and at different angles of pipeline.

As indicated in Fig. 5, the pressure is higher at the initial time due to the instantaneous closure valve. The pressure distribution oscillation based on the properties of transmission and reflection of pressure waves provoked by the instantaneous closure valve at the downstream end. At this stage, the pressure has built up due to the back flow at the downstream end of the pipeline. For natural gas, the maximum pressure reached to $34.23 \mathrm{bar}$ at horizontal pipeline and $35.48 \mathrm{bar}$ at inclined pipeline $\theta=15^{\circ}$. For hydrogen-natural gas mixture $\phi=0.25, \phi=0.5$ and $\phi=0.75$, the maximum pressure reached to $36.47 \mathrm{bar}, 38.83 \mathrm{bar}$ and $41.35 \mathrm{bar}$ at horizontal pipeline and $37.8 \mathrm{bar}, 40.25 \mathrm{bar}$ and $42.85 \mathrm{bar}$ at inclined pipeline $\theta=15^{\circ}$, respectively. For hydrogen, the maximum pressure reached to $44.12 \mathrm{bar}$ and $45.70 \mathrm{bar}$ at horizontal pipeline and inclined pipeline $\theta=15^{\circ}$, respectively.

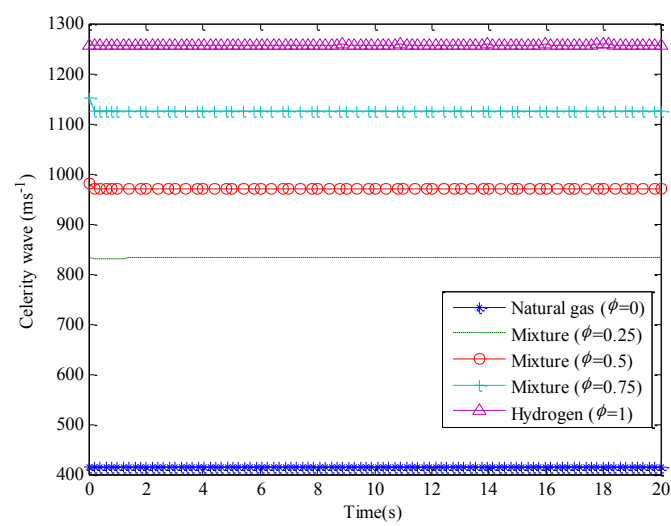

(a) Horizontal pipeline $\theta=0^{\circ}$

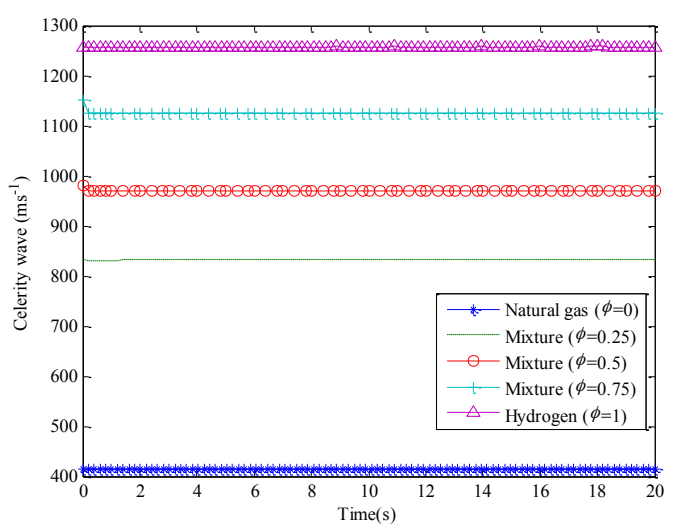

(b) Inclined pipeline $\theta=15^{\circ}$

Fig. 6 Celerity wave of hydrogen-natural gas mixture $\phi=0, \phi=0.25$, $\phi=0.5, \phi=0.75$ and $\phi=1$.

Fig. 6 presents the celerity wave distribution as a function of time with a sudden closure valve at leakage $X_{L}=L / 3$ for different mass ratio of hydrogen-natural gas mixtures $\phi$ at horizontal pipeline and different angles $\theta=15^{\circ}$. As seen in Fig. 6, leakage occurs at $t=0.81 \mathrm{~s}$ and the celerity wave are $414.1 \mathrm{~ms}^{-1}, 831.7 \mathrm{~ms}^{-1}$, $970.3 \mathrm{~ms}^{-1}, 1125.0 \mathrm{~ms}^{-1}$ and $1257.0 \mathrm{~ms}^{-1}$ for mass ratio of gas mixture at $\phi=0, \phi=0.25, \phi=0.5, \phi=0.75$ and $\phi=1$, respectively. The higher values of celerity wave is hydrogen and the lower is natural gas. It is observed that when the angle of pipeline increased to $\theta=15^{\circ}$, the obtained results are remain similar. From the results obtained in Fig. 6, noted that the celerity wave is not 
dependence to the inclination angle when the $\theta$ increased. Since the celerity wave results are used to calculate the leak location, thus the results of positions of leak location are remain similar, means that the inclination angle of pipeline $\theta$ does not affected to the leak location.

Numerical results on the effect of the mass ratio of a gas mixture of the transient pressure and celerity waves due to the inclined pipeline show that the leakage occurs at about $200 \mathrm{~m}$ and it can be denoted by $X_{L}=L / 3$. This leak location is detecting based on the pressure dropped results and these results are assumed as an estimated point. Referring to Figs. 5 and 6, the leak location and leak discharge of hydrogen-natural gas can be calculated where the leakage point $\Delta t$ equals to $0.81 \mathrm{~s}$ for leak occurring at $X_{L}=L / 3$. By using equation (38), the leak location of hydrogen-natural gas mixture at $\phi=0.5$ can be calculated as follows:

$$
X_{L}=600-\frac{0.81 \times 970.3}{2} \approx 207 \mathrm{~m}
$$

Table 1 presents the calculated values of the leak location for natural gas $\phi=0$, hydrogen-natural gas mixture at $\phi=0.25, \phi=0.5$, $\phi=0.75$, and hydrogen $\phi=1$ where the leak position $X_{L}=L / 3$ at horizontal pipeline. Since the results of celerity wave are remain similar for inclined pipeline at $\theta=15^{\circ}$, the position of leak locations are also do not affected. The leak locations for hydrogen-natural gas mixture are calculated based on the analysis of celerity wave results as indicated in Fig. 5. Based on Table 1, the leak location is determined to be occurred shorter for hydrogen-natural gas mixture at $\phi=0.75$ and hydrogen. At this point, the leakage occurs before our estimation point. For natural gas and hydrogen-natural gas mixture at $\phi=0.25$, and $\phi=0.5$, the leak occurs longer our estimation point $200 \mathrm{~m}$.

Table 1 Leak location of hydrogen-natural gas mixture at horizontal pipeline.

\begin{tabular}{ccc}
\hline Types of gas & Mass ratio $(\boldsymbol{\phi})$ & Leak location $(\mathbf{m})$ \\
\hline Natural gas & 0 & 432.3 \\
Hydrogen gas mixture & 0.25 & 264.5 \\
& 0.50 & 207.1 \\
Hydrogen & 0.75 & 144.4 \\
\hline
\end{tabular}

The amount of leakage discharge $Q_{L}$ can be calculated from equation (39). These results are obtained by analyzing the plots of Figs. 5 and 6 . The amount of leakage discharge of hydrogen-natural gas mixture at $\phi=0.5$, located at $X_{L}=L / 3$ in a horizontal pipeline is calculated as:

$$
Q_{L}=\frac{0.13}{970.3}\left(1.19 \times 10^{5}\right)=15.94 \mathrm{~kg} / \mathrm{s}
$$

Table 2 represents the amount of leak discharge for nonisothermal flow of natural gas $\phi=0$, hydrogen-natural gas mixture at $\phi=0.25, \phi=0.5$, and hydrogen $\phi=1$ where the leak position $X_{L}=L / 3$ with different angles of pipeline $\theta$. From Table 2, the results obtained clearly show that the amount of leak discharge increases when the inclination angle $\theta$ increases, while decreases when mass ratio of hydrogen-natural gas mixture $\phi$ are increases. To reduce the amount of leak discharge, the inclination angle of the pipeline should be reduced and further. Based on the results in Tables 1 and 2, the mass ratio of hydrogen to natural gas should not be more than 0.5 to ensure that leakage does not occur before the calculated leak position.
Table 2 Amount of leak discharge of hydrogen-natural gas mixtures.

\begin{tabular}{cccc}
\hline $\begin{array}{c}\text { Mass ratio } \\
(\boldsymbol{\phi})\end{array}$ & $\begin{array}{c}\text { Pipe angles } \\
(\boldsymbol{\theta})\end{array}$ & $\begin{array}{c}\left(\Delta \boldsymbol{P}_{\mathbf{0}}-\Delta \boldsymbol{P}_{\mathbf{I}}\right) \\
\left(\mathbf{N} / \mathbf{m}^{2}\right)\end{array}$ & $\begin{array}{c}\text { Leak discharge } \\
(\mathbf{k g} / \mathbf{s})\end{array}$ \\
\hline 0 & $0^{\circ}$ & $0.76 \times 10^{5}$ & 23.86 \\
& $15^{\circ}$ & $0.82 \times 10^{5}$ & 25.75 \\
0.25 & $0^{\circ}$ & $1.31 \times 10^{5}$ & 20.56 \\
& $15^{\circ}$ & $1.42 \times 10^{5}$ & 22.28 \\
0.5 & $0^{\circ}$ & $1.19 \times 10^{5}$ & 15.94 \\
& $15^{\circ}$ & $1.38 \times 10^{5}$ & 18.49 \\
0.75 & $0^{\circ}$ & $1.21 \times 10^{5}$ & 13.98 \\
& $15^{\circ}$ & $1.35 \times 10^{5}$ & 15.60 \\
1 & $0^{\circ}$ & $1.07 \times 10^{5}$ & 11.07 \\
& $15^{\circ}$ & $1.22 \times 10^{5}$ & 12.62 \\
\hline
\end{tabular}

\section{CONCLUSION}

The present investigation focuses on the development of a mathematical model for transient non-isothermal flow of hydrogennatural gas mixture in an inclined pipeline. This mixture is considered as hydrogen is often added to natural gas to enhance the latter's burning capacity, and because hydrogen needs to be transported in the same pipeline as natural gas due to its storage problem and to reduce transportation cost. The mathematical model developed took into consideration the effect of the mass ratio of gas mixture. The gas mixture was assumed to be homogeneous and the transient pressure wave was created by the sudden or instantaneous closure of a downstream shut-off valve to ensure the attainment of minimum pressure at the downstream end within a short time. The boundary conditions imposed were under the assumption that a reservoir exists at the upstream and a sudden closure valve was at the downstream. The governing equations consist of non-linear partial differential equations of unsteady, compressible and non-isothermal one dimensional flow. They were numerically solved using the ROM technique, which had not been previously applied on non-isothermal models involving a gas mixture. The transient pressure wave analysis was adopted to calculate the leak location and leak discharge. Specifically, the iron pipeline was taken to be $0.4 \mathrm{~m}$ in diameter, $600 \mathrm{~m}$ long, mass flow $Q_{0}=55 \mathrm{~kg} / \mathrm{s}$ at a static temperature $T=15^{\circ} \mathrm{C}$ and an absolute pressure $P=35$ bar. Numerical results on the effect of the mass ratio of a gas mixture of the transient pressure and celerity waves due to the inclined pipeline show that the leakage occurs at about $200 \mathrm{~m}$. It is observed that an increase in the mass ratio $\phi$ leads to an increase in the pressure and celerity wave, while the leak location and amount of leak discharge decrease. It is found that the mass ratio of hydrogen to natural gas should not be more than 0.5 to ensure that leakage does not occur before the estimated leak position. It is also observed that an increase in the inclination angle $\theta$ increases the pressure drop and leak discharge, but the celerity wave and the leak location do not seem to be affected.

\section{ACKNOWLEDGEMENT}

This work was financially supported by Universiti Teknologi Malaysia (UTM), Ministry of High Education (MOHE) and STEM Grant with vote no. A. J091002.5600.07397, Research University Grant Scheme, are gratefully acknowledged. 


\section{REFERENCES}

Agaie, B. G. (2014). Numerical Computation of Transient Hydrogen Natural Gas Mixture in a Pipeline Using Reduced Order Modelling. Doctor Philosophy, Universiti Teknologi Malaysia, Skudai.

Agaie, B. G. and Amin, N. (2014). The Effect of Water Hammer on Pressure Oscillation of Hydrogen Natural Gas Transient Flow. Applied Mechanics and Materials. 554: 251-255.

Agaie, B. G., Khan, I., Alshomrani, A. S. and Alqahtani, A. M. (2017). Reduced-Order Modelling for High-Pressure Transient Flow of HydrogenNatural Gas Mixture. The European Physical Journal Plus. 132: 234.

Bade, S. O. and Karim, G. A. (1999). Hydrogen as Additive to Methane for Sparking Ignition Engine Applications. International Journal for Hydrogen Energy. 24(6): 1-9.

Behbahani-Nejad, M. and Shekari, Y. (2008). Reduced Order Modeling of Natural Gas Transient Flow in Pipelines. International Journal of Engineering and Applied Sciences. 5(1): 148-152.

Behbahani-Nejad, M. and Shekari, Y. (2010). The Accuracy and Efficiency of a Reduced-Order Model for Transient Flow Analysis in Gas Pipelines. Journal of Pertoleum Science and Engineering. 73(1-2): 13-19.

Behbahani-Nejad, M., Haddadpour, H. and Esfahanian, V. (2004). Reduced Order Modeling of Unsteady Flows Without Static Correction Requirement. $24^{\text {th }}$ International Congress of the Aeronautical Sciences, ICAS. 29 August-3 September, 2004. Yokohama, Japan, 1-8.

Cheng, R. K., Littlejohn, D., Strakey, P. A. And Sidwell, T. (2009). Laboratory Investigation of a Low-Swirl Injection $\mathrm{H}_{2}$ and $\mathrm{CH}_{4}$ at Gas Turbine Conditions. Proceedings of the Combustion Institute. 32(2): 3001-3009.

Chiesa, P., Lozza, G. and Mazzocchi, L. (2001). Using Hydrogen as Gas Turbine Fuel. Journal of Engineering for Gas Turbines and Power. 127: $1-8$.

Veneri, O. (2011). Hydrogen as Future Energy Carrier. In Corbo, P., Migliardini, F. and Veneri, O (Eds.) Hydrogen Fuel Cells for Road Vehicles, Green Energy and Technology. 33-70. London: SpringerVerlag.

Elaoud, S. and Hadj-Taïeb, E. (2008). Transient Flow in Pipelines of HighPressure Hydrogen Natural Gas Mixtures. International Journal of Hydrogen Energy. 33(18): 4824-4832.

Elaoud, S. and Hadj-Taïeb, E. (2009). Leak Detection of Hydrogen Natural Gas Mixtures in Pipes Using the Pressure-Time Transient Analysis. Ecologic Vehicles Renewable Energies, EVER. 11-13 April, 2009. Monte Carlo, Monaco, 1-9.

Elaoud, S., Abdulhay, B. and Hadj-Taïeb, E. (2014). Effect of Hydrogen Injection into Natural Gas on the Mechanical Strength of Natural Gas Pipelines during Transportation. Archives of Mechanics. 66(4): 269-286.

Elaoud, S., Hadj-Taïeb, L. and Hadj-Taïeb, E. (2010). Leak Detection of Hydrogen Natural Gas Mixtures in Pipes Using the Characteristics Method of Specified Time Intervals. Journal of Loss Prevention in the Process Industries. 23: 637-645.

Giles, M. (1983). Eigenmode Analysis of Unsteady One-Dimensional Euler Equations. Hampton, Virginia: Institute for Computer Application in Science and Engineering NASA Langley Research Center.
Hall, K. C. (1994). Eigenanalysis of Unsteady Flows about Airfoils, Cascades, and Wings. The American Institute of Aeronautics and Astronautics (AIAA) Journal. 32(12): 2426-2432.

Hoeseldonackx, D. and D‘haeseleer, W. (2011). Concrete Transition Issues towards a Fully-Fledged Use of Hydrogen as an Energy Carrier: Methodology and Modelling. International Journal for Hydrogen Energy. 34: $1-16$.

Hoffmann, K. A. and Chiang, S. T. (2000). Computational Fluid Dynamics for Engineers Volume I. $4^{\text {th }}$ Edition. Wichita, Kansas USA: A publication of Engineering Education System.

Lydell, B. O. Y. (2000). Pipe Failure Probability-The Thomas Paper Revisited. Reliability Engineering and System Safety. 68: 1-11.

Michael, R. and Earl, D. (1996). Reduced Order Euler Equations for Unsteady Aerodynamic Flows-Numerical Techniques. AIAA $34^{\text {th }}$ Aerospace Sciences Meeting and Exhibit. 15 - 18 January 1996. Reno, NV: American Institute of Aeronautics and Astronautics, 34(8): 1578-1583.

Peet, T., Sagaut, P. and Charron, Y. (2009). Pressure Loss Reduction in Hydrogen Pipelines by Surface Restructuring. International Journal of Hydrogen Energy. 34(21): 8964-8973.

Sierens, R. and Rosseel, E. (2000). Variable Composition Hydrogen/Natural Gas Mixtures for Increased Engine Efficiency and Decreased Emissions. Journal of Engineering for Gas Turbines and Power. 122: 1-6.

Subani, N. Amin, N and Agaie, B. G. (2017). Leak Detection of NonIsothermal Flow of Hydrogen-Natural Gas Mixture. Journal of Loss Prevention in the Process Industries. 48: 244-253.

Subani, N. and Amin, N. (2015). Analysis of Water Hammer with Different Closing Valve Laws on Transient Flow of Hydrogen-Natural Gas Mixture. Abstract and Applied Analysis. 1-12.

Subani, N., Amin, N. and Agaie, B. G. (2015). Hydrogen-Natural Gas Mixture Leak Detection Using Reduced Order Modelling. Applied and Computational Mathematics. 4 (3): 135-144.

Tabkhi, F., Azzaro-Pantel, C., Pibouleau, L. and Domenech, S. (2008). A Mathematical Framework for Modeling and Evaluating Natural Gas Pipeline Networks under Hydrogen Injection. International Journal of Hydrogen Energy. 33 (21): 6222-6231.

Turner, W. J. and Mudford, N. R. (1988). Leak Detection, Timing, Location and Sizing in a Gas Pipelines. Mathematical and Computer Modelling. 10(8): 609-627.

Uilhoorn, F. E. (2009). Dynamic Behaviour of Non-Isothermal Compressible Natural Gases Mixed with Hydrogen in Pipelines. International Journal of Hydrogen Energy. 34(16): 6722-6729.

Veziroglu, T. N. and Barbir, F. (1992). Hydrogen: The Wonder Fuel. International Journal of Hydrogen Energy. 17(6): 391-404.

Weber, M. and Perrin, J. (2008). Hydrogen Transport and Distribution. Hydrogen Technology. Berlin Heidelberg: Springer-Verlag. 17(6): 129149.

Wilkening, H. and Baraldi, D. (2007). CFD Modelling of Accidental Hydrogen Release from Pipelines. International Journal of Hydrogen Energy. 32: 2206-2215. 